\title{
Measuring postpartum uterine contractions during the third stage of labour: a pilot study, using a novel minimally invasive technique
}

\author{
Bamigboye Anthony Akin ${ }^{1 *}$, Hofmeyr George Justus ${ }^{1}$, Nikodem Vernice Cheryl ${ }^{2}$
}

${ }^{1}$ Effective Care Research Unit, University of the Witwatersrand, South Africa and University Teaching Hospital Ado Ekiti, Ado Ekiti, Nigeria;

${ }^{1}$ Effective Care Research Unit, Fort Hare/Eastern Cape Department of Health, Universities of Witwatersrand, Witwatersrand, Johannesburg;

${ }^{2}$ Director of Research and Innovation, University of Venda, Thohoyandou, South Africa.

E-mail: "bami@medinet.co.za; justhof@global.co.za; cnikodem@medinet.co.za

Received 5 June 2011; revised 30 August 2011; accepted 9 September 2011.

\begin{abstract}
Objectives: To determine the feasibility of measuring intra-uterine pressure prior to placental delivery, using a novel minimally invasive method. Design: A prospective exploratory physiological study. Methods: Thirty-six low risk women undergoing normal vaginal delivery were randomly allocated to four groups. Group A received $600 \mathrm{mcg}$ rectal misoprostol; group $B$ received 600 mcg oral misoprostol; group $C$ received 10 international units oxytocin intramuscularly after delivery of the anterior shoulder; group D received intramuscular oxytocin, 30 minutes after delivery of the baby. A calibrated catheter-tip intrauterine pressure transducer was used to measure the intrauterine pressure. This was introduced into the placenta via the umbilical vein, and recorded on a standard tocograph. Results: It was technically easy to introduce the catheter through the umbilical vessels in all mothers. On assessment of the tocograms, it was possible to interpret 95 out of 108 recordings $(88 \%)$. Conclusions: This study demonstrated the simplicity of a novel, minimally invasive method of measuring the duration and frequency of postpartum uterine contractions prior to delivery of the placenta.
\end{abstract}

Keywords: Postpartum Haemorrhage; Intrauterine Contractions

\section{INTRODUCTION}

Excessive maternal blood loss after childbirth is a leading cause of morbidity and mortality, particularly in births without skilled attendance and where injectable uterotonics are not readily available. In the prevention and management of postpartum hemorrhage, different uterotonics have been used. Apart from clinical trials, there is a need to describe the physiological effectiveness of uterine contractions, especially when new uterotonic agents are being investigated. The method used to date for in vivo measurements has been direct placement of a transducer into the uterine cavity [1]. This is an invasive procedure with the possibility of introducing infection from the lower genital tract into the uterus. In addition, because the catheter tip is not in a fluid medium, the accuracy of the measurements may be in doubt. Previous physiological study found some correlation between blood loss and uterine activity in women given intravenous oxytocin, intramuscular oxytocin-ergometrine, or oral misoprostol. Intrauterine pressure was measured with a pressure catheter inserted into the uterine cavity after delivery of the placenta [1,2].

We looked into the possibility of measuring uterine activity with various commonly used uterotonics viz: early and late oxytocin administration, and orally and rectally administered misoprostol. We are not aware of any previous studies measuring the uterine response to uterotonics administration prior to placental delivery, nor use of the umbilical vein for access to the intrauterine space. The measurement of uterine activity prior to placental delivery is relevant because of the recent completion of a large World Health Organization trial (>23,000 women receiving routine uterotonics), comparing controlled cord traction with allowing spontaneous delivery of the placenta (unpublished data).

The study objectives were to evaluate a novel, minimally invasive method of measuring intrauterine pressure prior to placental delivery; and to determine the uterine contraction pattern (duration and frequency) following administration of misoprostol and oxytocin. 


\section{WOMEN AND METHODS}

The study protocol was approved by the Committee for Human Research of the University of the Witwatersrand, Johannesburg South Africa. Thirty-six low risk women who were to deliver at a tertiary hospital setting in Johannesburg, South Africa were recruited and gave informed consent. They were randomly allocated during the third stage of labor to four groups using a computer generated random sequence, in sequentially labeled treatment packs.

GROUP A 600 mcg rectal misoprostol as the active uterotonic agent.

GROUP B. 600 mcg oral misoprostol as the active agent.

GROUP C. 10 International Units oxytocin intramuscularly on delivery of the anterior shoulder. .

GROUP D. 10 International Units oxytocin 30 minutes after delivery.

A Hewlett Packard cardiotocogram monitor was activated to run the paper strip at $3 \mathrm{~cm}$ per minute. The tocogram pressure was zeroed after connection of a transducer tipped pressure catheter. Calibration was effected at $0 \mathrm{~cm}$ and $60 \mathrm{~cm}$ of water by immersion of the transducer in a $60 \mathrm{~cm}$ sterile water column. The time of delivery of the baby was noted. At delivery, with the placenta in situ, the pressure tip catheter was fed through the umbilical vein into the placental circulation and a soft clamp used to hold the catheter in place in the umbilical cord and prevent blood loss from the placental vasculature. The tocogram recording continued until either the placenta was spontaneously expelled or 30 minutes after insertion of the catheter.

The next in the sequence of refrigerated opaque envelopes containing the various randomized medications was opened to administer the contents, early or delayed oxytocin, and rectal or oral misoprostol. Oxytocin or ergometrine was to have been administered at any stage of the study if excessive uterine bleeding had occurred.

\section{RESULTS}

It was technically easy to insert the catheter through the umbilical vein in all mothers.

The baseline variables in terms of age and parity, for all the four groups of interventions, were similar (Table 1).

There was no difference between the various drugs in the three intervals of recordings as regards assessment for duration and/or frequency of uterine contractions. (Table 2)

In one woman, the placenta was delivered before the 30 minutes of recording time in the delayed oxytocin group, and one in the early oxytocin group. A few uninterpretable recordings on the tocogram were found in all groups recordings, and are represented by variations in ' $n$ ' in Table 2.

In the first 10 minutes of recording, 97\% of tocograms, using the various oxytocics regimen, had interpretable frequency and or duration of uterine contractions. The rates were $83 \%$ in the second and the last 10 minutes.

\section{DISCUSSION}

We have described an innovative method for physiological study of intrauterine pressure with the placenta in situ, with the catheter transducer introduced via the umbilical vein. The advantage of this approach may be a reduction in risk of introducing infections from the lower genital tracts into the raw, postpartum endometrium, and the inconvenience to the mother of direct intrauterine catheter placement. The procedure is simple and reproducible, as shown in the outcome for the different uterotonics used, in this exploratory trial.

\section{CONCLUSIONS}

This pilot study demonstrated the ease of a novel, minimally invasive method of postpartum intrauterine pressure measurement prior to delivery of the placenta. The benefits of introducing the pressure catheter within the umbilical vein were to avoid the discomfort and possible

Table 1. Baseline data expressed as median and range.

\begin{tabular}{|c|c|c|c|c|c|c|c|c|c|}
\hline & \multicolumn{2}{|c|}{ Oral Misoprostol } & \multicolumn{2}{|c|}{ Rectal Misoprostol } & \multicolumn{2}{|c|}{ Earily Oxytocin } & \multicolumn{2}{|c|}{ Late Oxytocin } & \multirow{2}{*}{ P value } \\
\hline & $\mathrm{N}$ & Median (rang) & $\mathrm{N}$ & Median (rang) & $\mathrm{N}$ & Median (rang) & $\mathrm{N}$ & Median (rang) & \\
\hline Age & 9 & $23(18-37)$ & 9 & $23(18$ - 37) & 9 & $23(18-43)$ & 9 & $27(18-32)$ & 0.400 \\
\hline Parity & 9 & $1(1-6)$ & 9 & $1(1-2)$ & $8^{*}$ & $1.5(1-40)$ & 9 & $2(1-4)$ & 0.258 \\
\hline
\end{tabular}

*Missing data.

Table 2. Interpretable tocogram of frequency and duration of contractions, using different oxytocic regimens during different time intervals post delivery.

\begin{tabular}{|c|c|c|c|c|c|c|c|c|c|c|}
\hline \multirow{2}{*}{$\begin{array}{l}\text { Frequency and/or } \\
\text { duration of } \\
\text { contraction }\end{array}$} & \multicolumn{2}{|c|}{ Oral Misoprostol, n = 9} & \multicolumn{2}{|c|}{ Rectal Misoprostol, n = 9} & \multicolumn{2}{|c|}{ Early Oxytocin, n = 9} & \multicolumn{2}{|c|}{ Late Oxytocin, $\mathrm{n}=9$} & \multicolumn{2}{|c|}{ Total } \\
\hline & $\mathrm{n}$ & $\%$ & $\mathrm{~N}$ & $\%$ & $\mathrm{~N}$ & $\%$ & $\mathrm{n}$ & $\%$ & $\mathrm{n}$ & $\%$ \\
\hline First 10 minutes & 9 & 100 & 9 & 100 & 8 & 88.9 & 9 & 100 & $35 / 36$ & 97 \\
\hline Second 10 minutes & 7 & 77.8 & 9 & 100 & 7 & 77.8 & 7 & 77.8 & $30 / 36$ & 83 \\
\hline Third 10 minutes & 7 & 77.8 & 8 & 88.9 & 8 & 88.9 & 7 & 77.8 & $30 / 36$ & 83 \\
\hline
\end{tabular}


introduction of infection associated with direct placement of a pressure catheter into the uterine cavity.

\section{REFERENCES}

[1] Chong, Y.S., Chua, S., El-Refaey, H., Choo, W.L., Chanrachakul, B., Tai, B.C., Rodeck, C. and Arulk-umaran, S. (2001) Postpartum intrauterine pressure studies of the uterotonic effect of oral misoprostol and intramuscular syntometrine. An International Journal of Obstetrics and Gynaecology, 108, 41-47.

[2] Choo, W.L., Chua, S., Chong, Y.S., Vanaja, K., Oei, P.L., Ho, L.M., Roy, A.C. and Arulkumaran, S. (1998) Correlation of change in uterine activity to blood loss in the third stage of labour. Gynecology and Obstetrics Investigation, 46, 178-180. doi:10.1159/000010028 